\title{
Intraoperative finding non-recurrent inferior laryngeal nerve, critical step of thyroid surgery, literature review, and case report
}

\author{
Ediel O. Dávila-Ruiz ${ }^{1 *}$, Roberto A. García-Manzano ${ }^{1}$, Nallely Y. Martínez-Santiago ${ }^{2}$, and \\ Daniel A. Bracamontes-Rosas ${ }^{3}$ \\ ${ }^{1}$ Department of General Surgery, Hospital Regional de Alta Especialidad de Oaxaca, Secretaria de Salud, San Bartolo Coyotepec, Oaxaca; \\ ${ }^{2}$ Department of General Surgery, Hospital General Sur Puebla "Dr Eduardo Vásquez Navarro", Puebla; ${ }^{3}$ Head Surgical Oncology Service, Hospital \\ Regional de Alta Especialidad de Oaxaca, Secretaria de Salud Oaxaca, San Bartolo Coyotepec, Oaxaca. Mexico
}

\begin{abstract}
Introduction: Non-recurrent laryngeal nerve is a relatively rare finding occurring in $0.3 \%-0.5 \%$ of people. Other authors estimate that prevalence was $0.7 \%$. Observation: We present a case of a female 48 years old who underwent hemithyroidectomy due to a thyroid nodule, identifying the presence of a non-recurring vertical variant laryngeal nerve. Discussion: The importance lies in the knowledge of the anatomical variations of the inferior laryngeal nerve (ILN). Since unilateral laryngeal paralysis is a complication associated with thyroidectomy, which is globally between 5 and $8 \%$ (transient) and 1 and $3 \%$ (permanent). The ILN should always be identified and meticulous surgical technique is necessary. Conclusions: ILN dissection should be guided by neurostimulation, if not counted, the recommendation that always should be followed is not to dissect structures until the laryngeal nerve is correctly identified.
\end{abstract}

Key words: Non-recurrent laryngeal nerve. Thyroidectomy. Injury recurrent laryngeal nerve. Anatomical variations inferior laryngeal nerve.

\section{Introduction}

According to GLOBOCAN 2018 thyroid cancer in the United States, it is the $8^{\text {th }}$ most common cancer with an incidence of 60,845 (18.6) and mortality of 2090 (0.64), its the $6^{\text {th }}$ most common cancer in Mexico, with an incidence of $12,122(9.3 \%)$ and mortality of 881 $(0.67 \%)$, worldwide thyroid cancer is $11^{\circ}$ with the incidence of $567,233(7.4)$ and mortality of $41,071(0.54)^{1}$.

In recent years, thyroid pathology has increased (benign/malignant); all this has led to increased interventions in the anterior neck region, as thyroidectomy, hemitiroidectomy, subtotal thyroidectomy, radical neck dissection.

Therefore, the identification efficiency of structures of vital importance in the neck should be increased: superior and inferior laryngeal nerve (ILN), vascular structures, and parathyroid glands.

The inferior laryngeal nerve (ILN) or recurrent laryngeal nerve (RLN), innervates the intrinsic muscles of the larynx including the posterior cricoarytenoid muscles which are responsible for vocal cord abduction and adduction, the RLN do not innervates the cricothyroid. RLN provides sensory fuction, involves the subglottic region.

During thyroid surgery, injury to the RLN is the major iatrogenic cause of vocal fold paralysis. Unilateral laryngeal paralysis is globally between 5 and $8 \%$ (transient) and 1 and $3 \%$ (permanent). After total thyroidectomy, the incidence of RLN injury uni or bilateral occurs around $4.3 \%$ (2.4\% transient and $1.3 \%$ definitive $)^{2}$.

\section{Correspondence:}

*Ediel O. Dávila-Ruiz

E-mail: drediel13@gmail.com
Date of reception: 20-01-2020

Date of acceptance: 13-03-2020

DOI: 10.24875/HGMX.20000015
Available online: 21-07-2020 Rev Med Hosp Gen Mex. 2020;83(3):131-134 www.hospitalgeneral.mx 0185-1063/@ 2020 Sociedad Médica del Hospital General de Mexico. Published by Permanyer. This is an open access article under the CC BYNC-ND license (http://creativecommons.org/licenses/by-nc-nd/4.0/). 


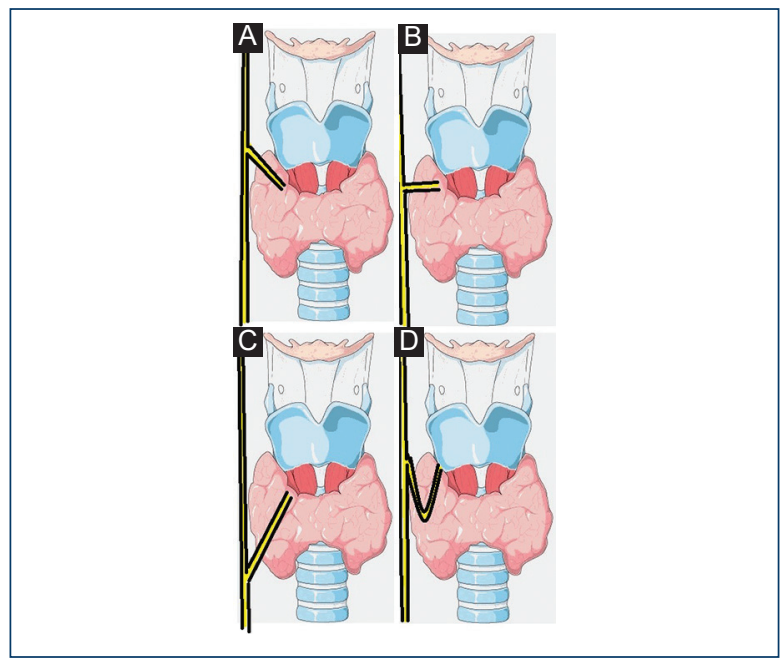

Figure 1. Four types of non-recurrent laryngeal nerve course. A: descending. B: vertical. C: ascending. D: V-shaped.

An awareness of RLN anatomical variations and meticulous surgical technique is necessary, non-RLN is a relatively rare finding occurring in $0.3 \%-0.5 \%$ of people, the first discovery of non-RLNs was in cadavers. Stedman reported a right-sided non-RLN in $1823^{3}$. Henry et al. (2017) report the pooled prevalence estimate was $0.7 \%$ (95\% confidence interval $[0.6-0.9)^{4}$. The NRLN was found to originate from the vagus nerve at or above the laryngotracheal junction in $58.3 \%$ and below it in $41.7 \%$. A right NRLN was associated with an aberrant subclavian artery in $86.7 \%$ of cases ${ }^{4}$.

Emmanouil et al, observation of the course of the NRLN 104 cases of non-RLN with confirmed vascular variants were reported. More specifically, $97.7 \%(n=$ 101) of cases involved a right and $2.3 \%(n=3)$ a left non-RLN. The most common concurrent vascular variant reported with a right non-RLN was an aberrant right subclavian artery $(97 \% ; n=98)^{5}$.

NRLN typically is classified into two types: Type I and II. Type I non-RLN arises directly from the cervical vagus and travels along the superior thyroid pedicle, while type II travels along the inferior thyroid artery, Type IIA runs more horizontally, parallel to the inferior thyroid artery trunk, whereas type IIB has a more ascending course, running inferior to the inferior thyroid artery trunk $k^{4,6}$.

Hong et al. describe the characteristic traveling patterns of non-RLN during surgery. Non-RLNs were identified on the right side in 15 patients who underwent thyroidectomy cases were classified according to four

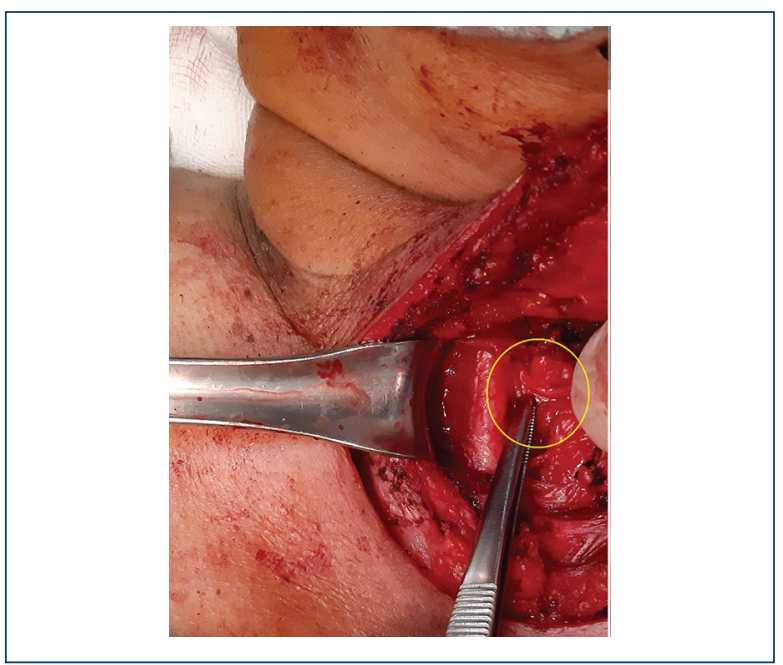

Figure 2. The right non-recurrent laryngeal nerve. Vertical variant.

non-RLN traveling pattern types: 1. descending - the nerve descends from the vagus nerve trunk; 2 . vertical - the nerve runs vertically to the cricothyroid joint; 3 . ascending - the nerve runs upward to the cricothyroid joint; and 4. V-shaped - the nerve takes a downward course and runs upward to the cricothyroid joint ${ }^{7}$ (Fig. 1).

The RLN is derived from the sixth branchial arch on embryogenesis, the fifth branchial arch regress, and the RLNs on both sides are around the structures from the fourth branchial arch (subclavian artery on the right side and the ligamentum arteriosum on the left). In the case of NRLN on the right side, there is a regression of the fourth arch, the right subclavian artery arises from the left side of the aorta and runs a retroesophageal course to the right neck base. Causing the right RLN not dragged down as the neck elongates, sometimes accompanied by other anomalies, situs invertus, aberrant subclavian artery, and absent ductus arteriosus ${ }^{6,8}$.

There are no symptoms or signs that indicate the possibility of a non-RLN pre-operatively. Some patients complained of mild dysphagia and a foreign body sensation ${ }^{6}$.

\section{Materials and methods}

An investigation made in PubMed using the terms Medical Subject Headings: RLN injuries and RLN and thyroidectomy/adverse effects OR thyroidectomy/complications and RLN/abnormalities or RLN/anatomy 


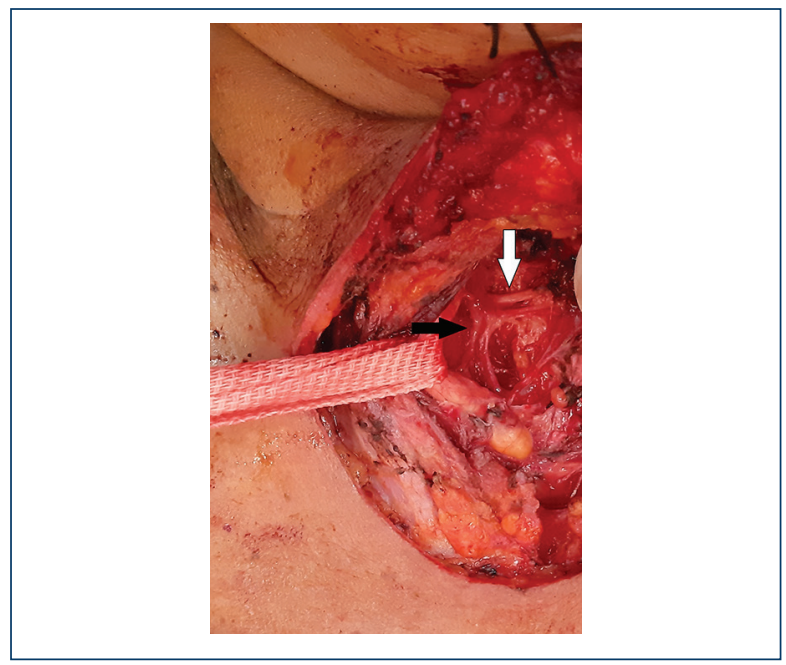

Figure 3. The right non-recurrent laryngeal nerve (white arrow), vagus nerve (black arrow).

obtaining ten articles related to the topic, selected for review, presenting the case of a patient with transoperative findings of non-RLN.

\section{Case presentation}

A 48-year-old female with diabetes and hypertension of 5 months diagnosis, no more antecedents, begins current condition 4 months ago with an increase in volume at the right neck muscle triangle, $3 \mathrm{~cm}$ in diameter, hard consistency, painful, an ultrasound reports a thyroid nodule dependent on the right lobe of $35 \times 22$ $\mathrm{mm}$ of component cystic, and hypervascular pattern by color Doppler. Surgical protocol for the approach of a solitary thyroid nodule, programming a right unilateral thyroidectomy.

Right hemitiroidectomy was performed with a Kocher approach, dissecting upper, and lower flap, separates midline, and proceeds to dissect prethyroid muscles. Thyroid was separated from the upper pedicle and the search for the right RLN, below the lower parathyroid of the same side, in the esophagotracheal groove.

The right RLN was not found so that a carotid sheath exploration is decided, to identify a possible anatomical variant, identifying the presence of a non-recurring vertical variant laryngeal nerve, in which the nerve runs vertically toward the cricothyroid joint (Fig. 2).

Once the nerve has been identified and with the certainty of not damaging any structure, the inferior pedicle was ligated and cut; the right lobe hemitoridectomy is finished with minimal bleeding quantification, $1 \mathrm{~h}$ surgical time (Fig. 3).

\section{Results}

Post-operative period patient refers only mild pain in the surgical approach, adequate tolerance of the oral diet, without complications, hypocalcemia data are not shown, with surgical wound with Kocher type approach without bleeding or dehiscence. No alterations in phonation or stridor were monitored externally through consultation. No functional repercussions at 30 days.

Pathology report: thyroid lobe with macro and microfollicular adenoma, moderate chronic thyroiditis, and vascular congestion. No evidence of malignancy in the examined tissue.

Not meriting further procedures.

\section{Discussion}

It is currently established that the identification of the nerve throughout its trajectory, with a systematic dissection, confers a lower risk of injury. RLN originates in the lower portion of the ambiguous nucleus of the spinal nerve, reaches the vagus nerve and joins in its intracranial portion before exiting through the jugular hole. It continues its path attached to the vagus nerve and separates when it has descended to the lower portion of the neck on the right side, forming a handle at level of the right subclavian artery and on left side in upper portion of the thorax in the aortic arch. Ascending through the tracheoesophageal groove, crossing the inferior thyroid artery, entering the larynx, once inside, together with the inner branch of the superior laryngeal nerve, forms the Galen's laryngeal loop, which serves for phonation, maintains the aerial pathway permeable during swallowing, breathing and innervation of the laryngeal mucosa, inferior to the vocal cords $^{9}$. If the presence of non-recurrent nerve is unknown the lesion is more likely, the risk of injury to the RLN is $1-2 \%$, while in contrast the risk of injury to a non-RLN skyrockets to $12.9 \%$, so the awareness of its existence and a correct surgical technique will prevent injuries of the nerve during cervical surgery ${ }^{10}$.

lacobone et al. (2015) showed that pre-operative ultrasonography to assess patients for an NRLN was extremely successful, with an accuracy of more than $98 \%{ }^{11}$.

Intraoperative neuromonitoring (NMIO) is used progressively more frequently, and the American Academy of Otolaryngology and Head and Neck Surgery recommends performing NMIO because: reduces time to identify the non-leukoreduced (NLR), the incidence of injuries and helps avoid bilateral injury, offers information on 
laryngeal nerve function at the end of surgery, with an accuracy of more than $95 \% 6,7,10,12$.

The Riddell criteria for the prevention of laryngeal paralysis should be followed: visual identification of the NLR in relation to the inferior thyroid artery, functional verification after the end of the intervention by electrical stimulation and routine and post-operative laryngoscopy (if necessary) ${ }^{2,12}$.

Pardal-Refoyo et al., in a meta-analysis, showed that the incidence of nerve injury with NM was lower than without NM $(2.4 \%, 1.55-3.5 \%]$ versus $5.18 \%, 2.53$ $8.7 \%]$ ), absolute risk reduction of $2.75 \% 2$.

\section{Conclusions}

In summary, the dissection of the laryngeal nerve should be guided by neurostimulation, if not counted, the recommendation that should always be followed is not to dissect structures until the laryngeal nerve is correctly identified, if there is doubt, the dissection of the carotid sheath to look for the vagus nerve is an acceptable maneuver. In addition, dissection and careful handle of tissues should always be a premise as in all surgical procedures.

\section{References}

1. International Agency for Research on Cancer, Cancer Today. Globocan 2018. Available from: http://www.gco.iarc.fr. [Last accessed on 2020 Feb 16].

2. Pardal-Refoyo JL, Ochoa-Sangrador C. Lesión bilateral del nervio laríngeo recurrente en tiroidectomía total con o sin neuromonitorización intraoperatoria. Revisión sistemática y metaanálisis. Acta Otorrinolaringol Esp. 2015;67:66-74.

3. Monahan TS. Nonrecurrent inferior laryngeal nerve. Vasc Endovasc Surg. 2010;45:90-1.

4. Henry BM, Sanna S, Graves MJ, Vikse J, Sanna B, Tomaszewsk IM, et al. The Non-recurrent laryngeal nerve: a meta-analysis and clinical considerations. Peer J. 2017:5:e3012.

5. Bakalinis E, Makris I, Demesticha T, Tsakotos G, Skandalakis P, Fillippo D. Non-Recurrent Laryngeal Nerve and Concurrent Vascular Variants: A Review, Acta Medica Academica 2018;47(2):186-92. doi: 10.5644/ ama2006-124.230

6. Kamani D, Potenza AS, Cernea CR, Kamani YV, Randolph GW. The nonrecurrent laryngeal nerve: anatomic and electrophysiologic algorithm for reliable identification. Laryngoscope. 2015;125:503-8.

7. Hong KH, Park HT, Yang YS. Characteristic travelling patterns of non-recurrent laryngeal nerves. J Laryngol Otol. 2014;128:534-9.

8. Forde R, Williams EW. The non-recurrent laryngeal nerve-a rare phenomenon which requires vigilance. West Indian Med J. 2015;64:3034.

9. Rojas M, Quijano Y, Luque-Bernal RM. Variaciones anatómicas del nervio laríngeo recurrente en una muestra de población colombiana. Rev. Fac. Med. 2016;64(2)207-13. doi: http://dx.doi.org/10.15446/revfacmed. v64n2.50643

10. Prat-Calero A, Cascales-Sánchez P, Martínez-Moreno A. Hallazgo intraoperatorio, de nervio laríngeo inferior no recurrente, durante intervención por carcinoma papilar de tiroides. Acta Otorrinolaringol Esp. 2017;68:62-3.

11. lacobone M, Citton M, Pagura G, Viel G, Nitti D. Increased and safer detection of nonrecurrent inferior laryngeal nerve after preoperative ultrasonography. Laryngoscope. 2015;125:1743-7.

12. Pardal-Refoyo JL, Arroyo-Domingo MM, Vargas-Yglesias E, Maza-Solano JM, Granell J, Martínez-Salazar JM, et al. Recomendaciones sobre el uso de la neuromonitorización en cirugía de tiroides y paratiroides. Acta Otorrinolaringol Esp. 2017;69:12. 